\title{
DYNAMICS OF THE ZODIACAL CLOUD
}

\author{
S. F. DERMO'T'T \\ Department of Astronomy, University of Florida, Gainesville, FL 32611, USA \\ R. S. (IONES \\ Observatório Nacional, Departamento de Astronomia, Rio de Janeiro, Brazil \\ D. D. DURDA. B. À. S. GUSTAFSON, S. JAYARAMAN and Y. L. XU \\ Department of Astronomy, University of Florida, Gainesville, FL 32611, USA \\ and \\ P. D. NICHOLSON \\ Department of Astronomy, Cornell University, Ithaca, NY 14853, USA
}

\begin{abstract}
Advances in infrared astronomy and in computing power have recently opened up an interesting area of the solar system for dynamical exploration. The survey of the sky made by The Infrared Astronomical Satellite (IRAS) in 1983 revealed the complex structure of the zodiacal dust cloud. We now know the inclination and nodes of the plane of symmetry of the cloud with respect to the ecliptic and we have evidence that the cloud is not rotationally symmetric with respect to the Sun. Of even more interest is the discovery by IRAS of prominent dust bands that circle the Sun in planes near-parallel to the ecliptic. In 1984, we suggested (Dermott et al., Nature, 312, 505-509) that the solar system dust bands discovered by IRAS are produced by the gradual comminution of the asteroids in the major Hirayama asteroid families. The confirmation of this hypothesis has involved: (1) The development of a new secular perturbation theory that includes the effects of Poynting-Robertson light drag on the evolution of the dust particle orbits; (2) The production of a new high resolution Zodiacal History File by IPAC (the Infrared Processing and Analysis Center at Caltech); (3) The development of the SIMUL code: a three-dimensional numerical model that allows the calculation of the thermal flux produced by any particular distribution of dust particle orbits. SIMUL includes the effects of planetary perturbations and PR drag on the dust particle orbits and reproduces the exact viewing geometry of the IRAS telescope. We report that these tools allow us to account in detail for the observed structure of the dust bands. They also allow us to show that there is evidence in the IRAS data for the transport of asteroidal dust from the main belt to the Earth by Poynting-Robertson light drag.
\end{abstract}

\section{Introduction}

Most previous attempts at modeling the zodiacal cloud have been based on finding a distribution of particle number density $n(\mathbf{r})$, where $\mathbf{r}$ is the heliocentric position vector, that satisfies the various observations. The number density function in these models is not derived from first principles, rather it is usually assumed to have the form

$$
n(r, \beta)=n_{0}(r / A U)^{-\nu} f(\beta)
$$

where $n_{0}$ is the particle number density at Earth orbit and $\beta$ is the heliocentric latitude (Giese and Kneißel, 1989). The models are heliocentric and rotationally symmetric and usually do not distinguish between the plane of symmetry of the cloud and that of the ecliptic. However, new spacecraft observations, particularly those by IRAS (the Infrared Astronomical Satellite), have resulted in a highly precise description of the cloud. Also, it is now known that the zodiacal cloud is not featureless: IRAS discovered circumsolar near-ecliptic bands of dust that appear to be related to the prominent Hirayama asteroid families (Dermott and 
Nicholson, 1989), suggesting that the asteroid belt as a whole is a significant source of interplanetary dust particles (IDPs). Levasseur and Blamont (1976) reported in the seventies that fluctuations in the intensity of the zodiacal light exist, some of which they were able to correlate with cometary debris. We consider that the quality of the new spacecraft observations demands a sophisticated approach to the modeling of the zodiacal cloud that is both more direct and more physically meaningful than previous methods.

Our aim is to use IRAS and other observations (Helios, COBE, and groundbased optical observations) to place constraints on the origin and evolution of interplanetary dust particles from source to sink. Our approach is to start with a postulated source of particles, either asteroidal or cometary, and then describe:

1. The orbital evolution of these particles due to Poynting-Robertson drag, using equations of motion that include light pressure and gravitational perturbations.

2. The collisional evolution of the particles and the variation of their sizefrequency distribution with heliocentric distance.

3. The thermal and optical properties of the particles and their variation with particle size.

We do not claim that all of this can be done from first principles. There are far too many unknowns involved, particularly in items 2 and 3. Rather, our approach is to parameterize these unknowns and then adjust them until our model matches the observations. We will then have some real information on the nature of the particles that, hopefully, can be tested by other means.

Other sources of information on the nature, origin and evolution of IDPs include (this list is far from complete): (1) The physical condition of micrometeorites. Flynn (1992) has reported on his analysis of the atmospheric heating of large micrometeorites and concluded that survival without melting demands a low relative velocity and that this favors an asteroidal source. (2) Spacecraft measurements of fluxes, masses and velocities: Grün (1992) has recently reported that measurements by the Galileo and Ulysses spacecraft of the variations of the dust particle fluxes with the orientations of the detectors indicate that the dust particle orbits are more consistent with an asteroidal than a cometary source. (3) Laboratory analysis of IDPs: Schramm et al.(1989) analyzed 200 interplanetary dust particles and concluded that $45 \%$ are probably cometary but $37 \%$ have characteristics (chemical alteration by liquid water) that suggest an asteroidal origin. The most valuable data will be obtained from the Cosmic Dust Collection Facility to be flown on Space Station Freedom. This facility will enable us to determine the orbits of the particles impacting the collectors and will give definitive information on their nature and origin.

\section{Orbital Evolution due to Poynting-Robertson Light Drag}

The most obvious feature of the zodiacal cloud that demands a dynamical explanation is the observation that the plane of symmetry of the cloud is inclined to 

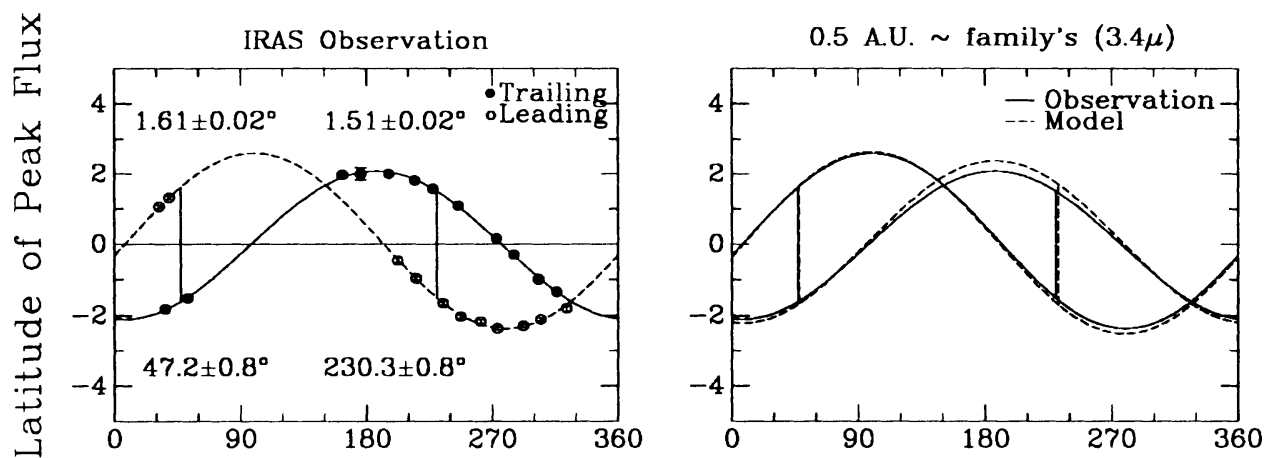

Fig. 1. (a) Variation of the ecliptic latitude (in degrees) of the peak background zodiacal emission with the ecliptic longitude of the Earth observed by IRAS in the $25 \mu m$ waveband at an elongation angle of $90^{\circ}$ in both the leading and the trailing directions. The numbers and vertical lines refer to the inclinations and the ascending and descending nodes of the clond. The points on the curves have error bars that are almost too small to be seen in this figure, testifying to the high quality of the IRAS observations. (b) Comparison of the IR $\Lambda S$ obscrvations shown in (a) with our model of the cloud based on asteroidal particles of $3.4 \mu \mathrm{m}$ radii whose orbits decay due to PR drag.

the ecliptic. The inclinations and nodes of the planes of symmetry of the background cloud and the dustbands are particularly robust diagnostic tools because their determination is unaffected by the calibration problems that plague the interpretation of the amplitudes of the signals seen in the various IRAS wavebands. We have learned how to exploit the IRAS observing sequence in order to coadd and process data in such way that the solar system signal is enhanced while the galactic noise is reduced. This has allowed us to make very precise determinations of the orientation of the cloud and of some of the other key parameters that describe the cloud's structure. Figure 1(a) shows the variation with the ecliptic longitude of the Earth of the ecliptic latitude of the peak flux observed by IRAS in the $25 \mu \mathrm{m}$ waveband. The elongation angle is precisely $90^{\circ}$ and we show the variations in both the leading and the trailing directions. (If the line of sight of the telescope on crossing the ecliptic points in the direction of the Earth's orbital motion, then we define that direction as the leading direction.) Those points in the Earth's orbit at which the latitudes of the peak fluxes in the leading and trailing directions are equal and opposite define the nodal longitudes, while the latitudes of the peaks at these nodal points give the inclinations.

The plane of symmetry of the zodiacal cloud is the plane about which, on average, the proper inclinations of the particle orbits precess. Its orientation is determined by the forced inclinations and nodes imposed on the dust particle orbits by the perturbations of the planets. These can be calculated using classical secular perturbation theory (Dermott et al., 1985, 1986, 1988), after allowance has been made for the effects of drag forces (Gomes and Dermott, 1992). The results of the 


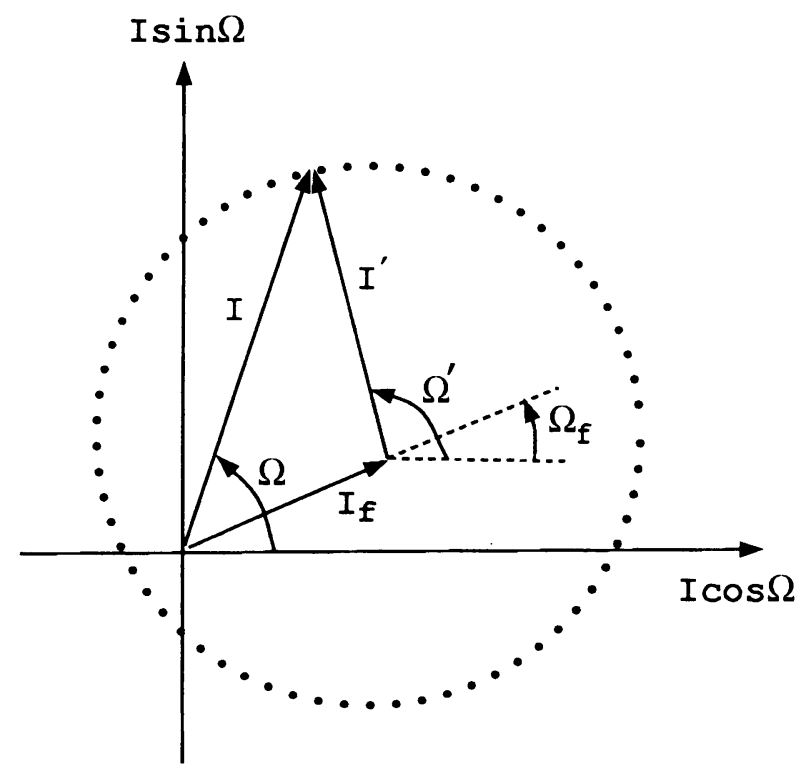

Fig. 2. Resolution of the inclination $(I)$ of a dust particle's orbit into its forced $\left(I_{f}\right)$ and proper $\left(I^{\prime}\right)$ components.

classical theory are summarized in Figure 2.

Consider the motion of a dust particle in the region of the asteroid belt. In the absence of drag forces, the semimajor axis of the particle is constant and the long-term variations in the orbital eccentricity and inclination are determined by the secular terms in the particle's disturbing function, that is, by those terms in the disturbing function that do not depend on any of the mean longitudes. If the eccentricities and inclinations are small, and we retain only the lowest order secular terms in the disturbing function, then the solutions of Lagrange's equations of motion for the eccentricity and pericenter variations are completely decoupled from the inclination and node variations. Both solutions are very similar. They both have simple vectorial representations and the distribution of orbits based on these solutions have correspondingly simple geometrical interpretations (Dermott et al., 1985).

The variation of the osculating inclination, $I$, is given by

$$
\begin{aligned}
& I \sin \Omega=I_{f} \sin \Omega_{f}+I^{\prime} \sin \left(\dot{\Omega}^{\prime} t+\Omega_{0}\right) \\
& I \cos \Omega=I_{f} \cos \Omega_{f}+I^{\prime} \cos \left(\dot{\Omega}^{\prime} t+\Omega_{0}\right)
\end{aligned}
$$

where the proper inclination, $I^{\prime}$, depends on the initial conditions. The forced inclination and node, $I_{f}$ and $\Omega_{f}$, imposed on the particle's orbit by the planets and the time rate of change of the particle's proper node, $\Omega^{\prime}$, are independent of all the orbital elements of the particle except its semimajor axis. The asteroids in a Hirayama family have slightly different semimajor axes and, hence, slightly different values of $\Omega^{\prime}$. After the initial formation of a family by some collision, the points 
representing the asteroids will spread out, on a timescale given by the differential precession rate, to form a near-perfect circle in the inclination vector diagram of the family - see Figure 2.

The forced inclination vector is not constant but varies with time according to

$$
\begin{aligned}
& I_{f} \sin \Omega_{f}=\sum_{l} \frac{\mu_{l}}{\left(\Omega^{\prime}-f_{l}\right)} \sin \left(f_{l} t+\gamma_{l}\right) \\
& I_{f} \cos \Omega_{f}=\sum_{l} \frac{\mu_{l}}{\left(\Omega^{\prime}-f_{l}\right)} \cos \left(f_{l} t+\gamma_{l}\right)
\end{aligned}
$$

where the $f_{l}$ are the eigenfrequencies of the solar system associated with the motions of the nodes of the planetary orbits and the $\mu_{l}$ are related to the amplitudes of the corresponding eigenmodes (Dermott et al., 1985; Dermott and Nicholson, 1989). Because the changes in the forced inclination vectors and the rotations of the proper inclination vectors at each time step depend only on the semimajor axis of the asteroid, they are the same for all the asteroids in a given family and thus those asteroids that are initially distributed on a circle in the vector diagram remain on a circle of the same radius even though the location of the center of that circle changes on a time scale comparable to that on which the asteroidal proper elements rotate about the circle's center. Similar expressions can be written for the variations of the osculating eccentricity. The concepts of forced and proper orbital elements that are useful in describing both the results of classical secular perturbation theory and the equilibrium distribution of the orbital elements of a cloud of particles, remain equally useful after the addition of drag forces to the equations of motion: only the method of their calculation changes (Gomes and Dermott, 1992).

The equation of motion of a particle of mass $m$ and geometrical cross-section $A$, moving with velocity $\mathbf{v}$ through a radiation field of energy flux density $S$, is given by

$$
m \dot{\mathbf{v}}=(S A / c) Q_{p r}[(1-\dot{r} / c) \hat{\mathbf{S}}-\mathbf{v} / c]
$$

where $\hat{\mathbf{S}}$ is a unit vector in the direction of the incident radiation, $\dot{r}$ is the particle's radial velocity and $c$ is the speed of light. The radiation pressure efficiency factor is given by

$$
Q_{p r} \equiv Q_{a b s}+Q_{s c a}(1-<\cos \alpha>)
$$

where $Q_{a b}$, and $Q_{s c a}$ are the efficiency factors for absorption and scattering, and $\langle\cos \alpha>$ accounts for the asymmetry of the scattered radiation (Burns et al., 1979). For a perfectly absorbing particle, $Q_{p r}$ is unity. The semimajor axis $a$ decays at rate given by

$$
\frac{1}{a}\left\langle\frac{d a}{d t}\right\rangle=-\frac{\left(\eta / a^{2}\right) Q_{p r}\left(2+3 e^{2}\right)}{\left(1-e^{2}\right)^{3 / 2}} \approx-\frac{2 \eta Q_{p r}}{a^{2}}
$$

(Wyatt and Whipple, 1950; Burns et al., 1979), where

$$
\eta=2.53 \times 10^{11} / \rho s
$$

for spherical particles in heliocentric orbits, assuming that the particle density $\rho$ and radius $s$ are in cgs units. Thus, to first order in $e, \dot{a}$ does not depend on 

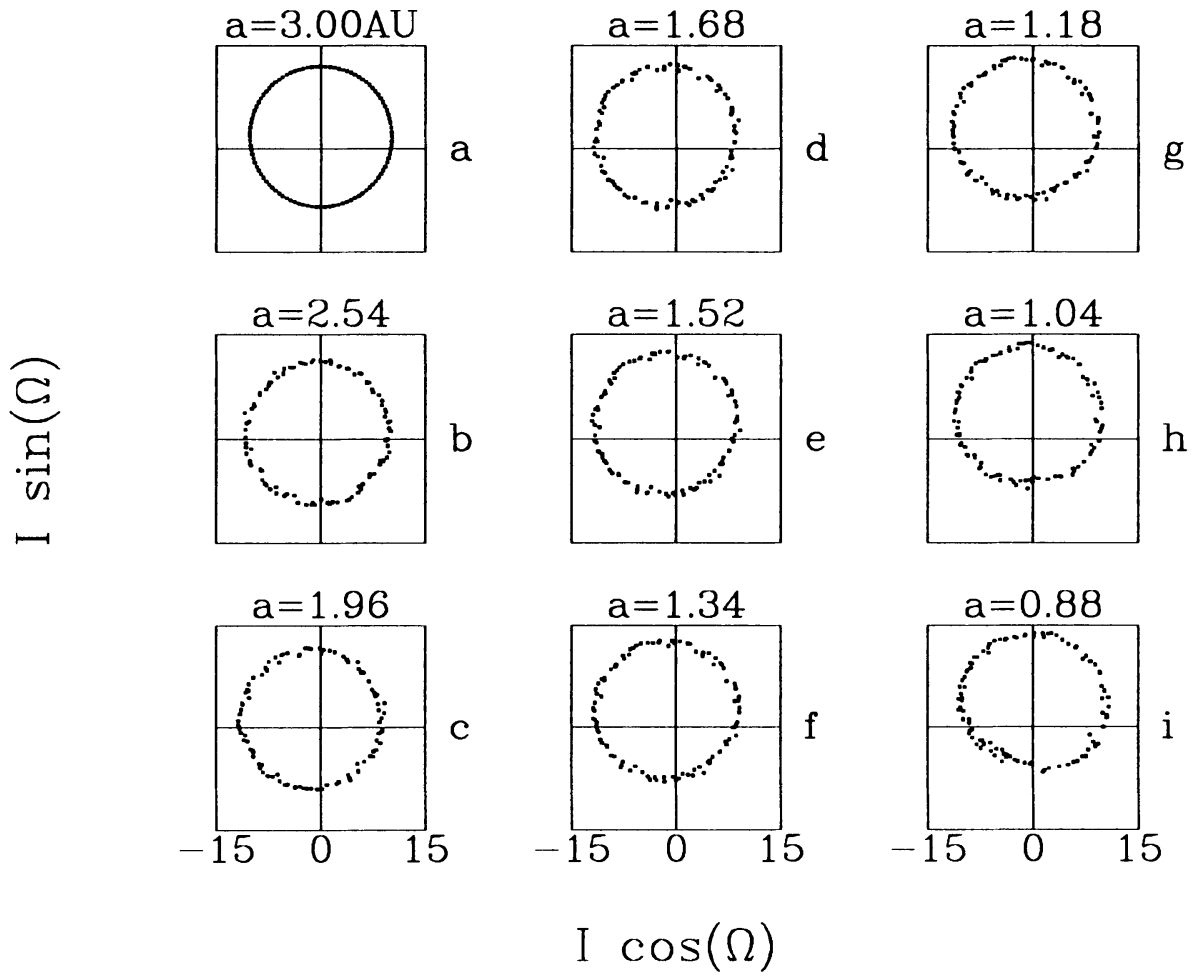

Fig. 3. Variation of the inclination vectors of an array of 100 particles of radii $3.4 \mu m$ and initial orbital elements similar to those of the Eos family asteroids. The panels $a-i$ show the distributions of the vectors in 1983 for various mean heliocentric distances in $A U$.

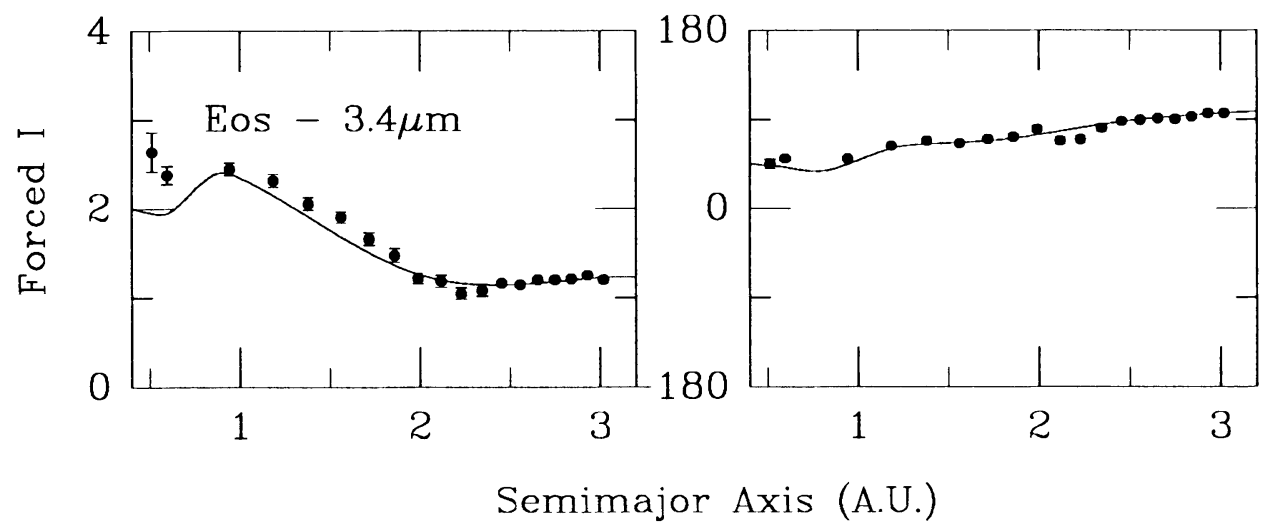

Fig. 4. Variation of (a) the forced inclination and (b) the longitude of the forced node with respect to the ecliptic of the Eos particles shown in Figure 3. 

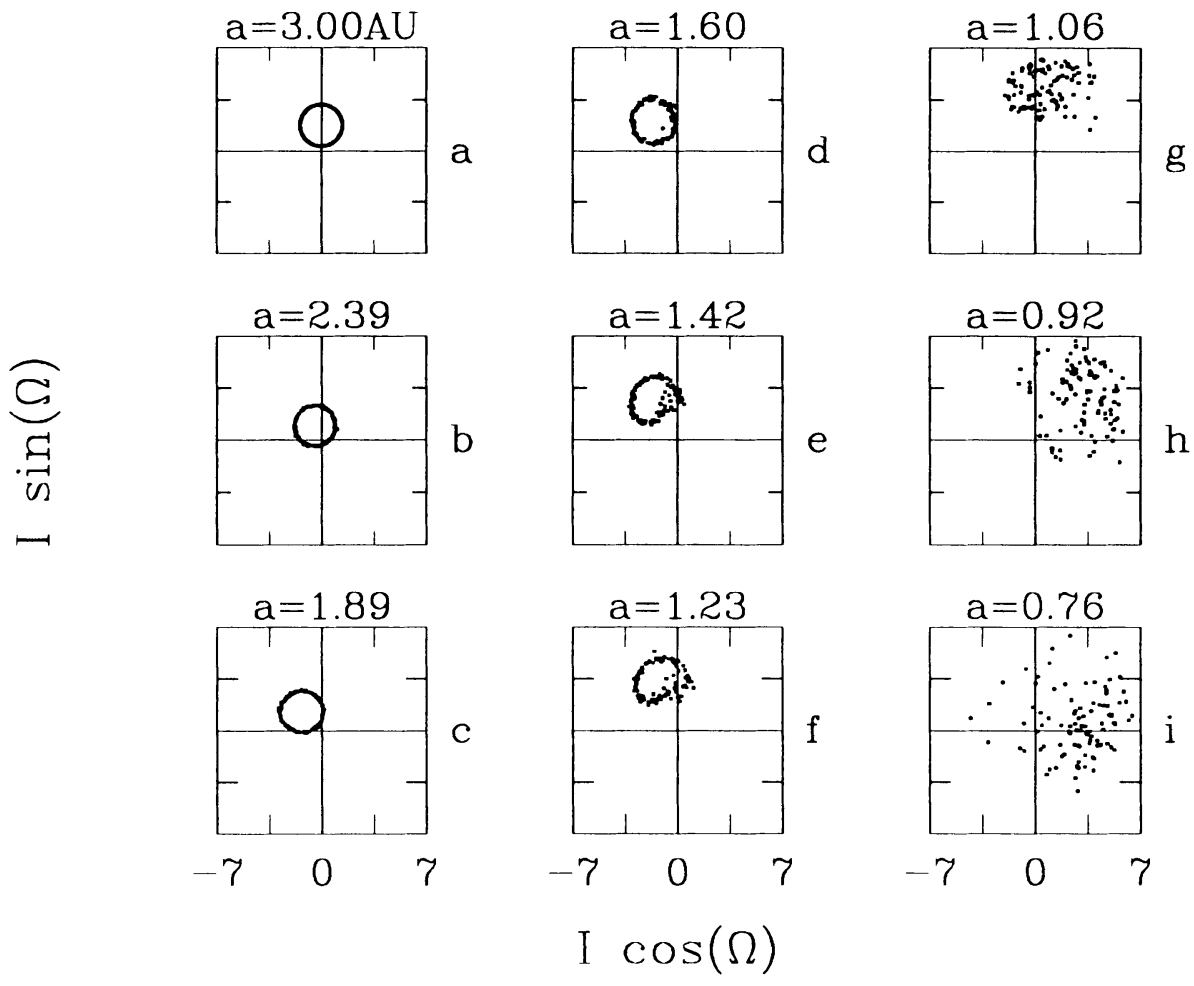

Fig. 5. Variation of the inclination vectors of an array of 100 particles of radii $3.4 \mu m$ and initial orbital elements similar to those of the Themis family asteroids. The pants $a-i$ show the distributions of the vectors in $\mathbf{1 9 8 3}$ for various mean heliocentric distances in $A U$.

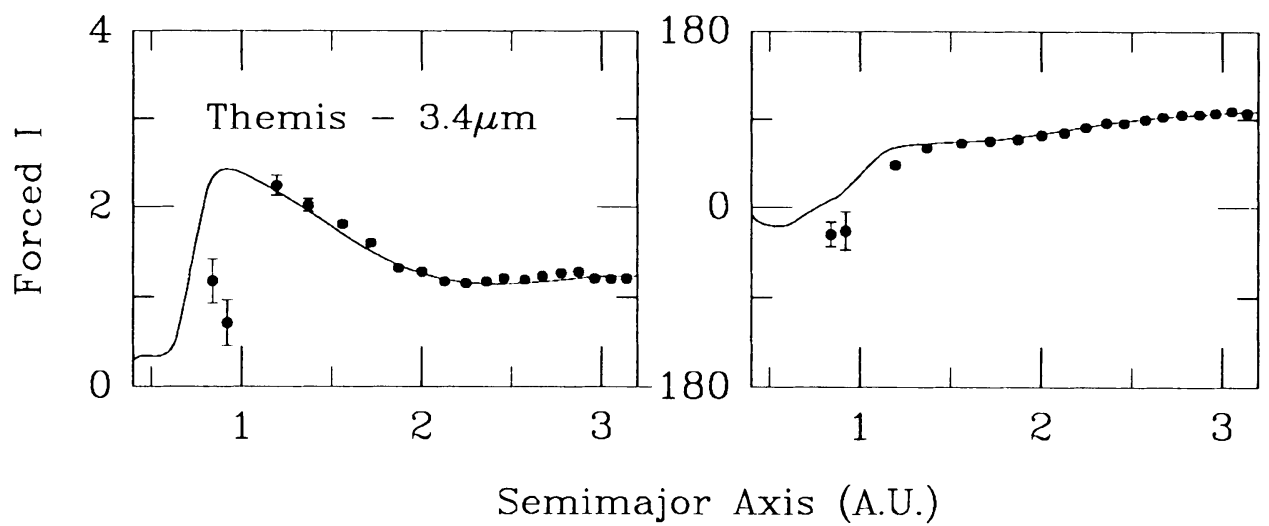

Fig. 6. Variation of (a) the forced inclination and (b) the longit ude of the forced node with respect to the ecliptic of the Themis particles shown in Figure 5. 
either $e$ or $I$, but it is directly proportional to the particle radius $s$. The orbits of asteroidal particles of radii $3 \mu \mathrm{m}$ decay on a timescale of $\sim 10^{5}$ years, not very much longer than the nodal precession period $\left(\sim 2 \cdot 10^{4}\right.$ years $)$.

The radiation forces act in the plane of the particle's orbit and thus have no direct effect on the particle's orbital inclination. The proper inclinations remain constant, apart from small changes produced by passage through the Kirkwood gaps and other orbit-orbit resonances. However, the forced inclinations vary with both particle size and heliocentric distance. If we consider a family of single-size particles, that is, particles with identical radii, that are initially distributed in the inclinationvector diagram on a circle of center determined by their common forced inclination, then, because these particles experience the same change in their semimajor axes at each time step in their orbital evolution, they will also experience the same change in their forced inclination vectors and thus they will remain on a common circle. The evolution of the center of this circle now depends on both the planetary perturbations and the drag rate, and is easily determined by numerical integration of the equations of motion.

Comparisons of the results obtained by integrating the truncated equations of motion given by secular perturbation theory and by integrating the full equations of motion associated with pointmass gravitational forces are shown in Figures 3-6. Each of the panels in Figures 3 and 5 shows the results of a separate numerical integration using the full equations of motion for $3.4 \mu m$ radii particles moving under the influence of PR drag, light pressure and direct gravitational forces due to Venus, Earth, Mars, Jupiter, Saturn, Uranus and Neptune. The semimajor axes above each panel are the mean semimajor axes of the particles at the end of the integration. In each case 100 particles were initially distributed on a perfect circle in the vector diagram. The center of the circle is given by the forced inclination of the parent asteroids at the starting epoch and that is determined by the initial osculating elements $e, I, \varpi$ and $\Omega$, of the planets. These were chosen such that at the end of each integration, the elements were those of the planets in 1983, the year of the IRAS observations. Similar considerations apply to the eccentricities. Thus, the elements shown in Figures 3 and 5 represent the distribution of the orbital elements of a cloud of asteroidal particles at various distances from the Sun at one instant in time, the year 1983 .

The points with error bars in Figures 4 and 6 were obtained from the mean centers of the circles of points in the panels shown in Figures 3 and 5 . The solid curves describe the variation of the forced inclination with heliocentric distance for the year 1983, obtained from numerical integration of the truncated equations of motion, that is, from the modified secular perturbation theory. The agreement between theory and the full integrations is excellent. Figure 3 shows the results obtained for particles from the Eos Hirayama family that have proper inclinations of $10.2^{\circ}$. Figure 5 shows the results obtained for particles from the Themis Hirayama family that have proper inclinations of $1.42^{\circ}$. The Eos particles remain sensibly on a circle, even after crossing the orbit of the Earth. However, the Themis particles with low inclinations suffer significant scattering on encountering Mars and major displacements on encountering Earth and Venus. Nevertheless, outside the orbit of the Earth, the centers of mass of the points in the panels remain close to the 
predicted curve.

The radiation forces act to decrease the particle's total orbital eccentricity at a rate given by

$$
\frac{1}{e}\left\langle\frac{d e}{d t}\right\rangle=-\frac{5 \eta Q_{p r}}{2 a^{2}\left(1-e^{2}\right)} \approx-\frac{5 \eta Q_{p r}}{2 a^{2}}
$$

These forces reduce both the proper and the forced eccentricities of the particles, but passage through secular resonances acts to increase these eccentricities. We do not discuss these results here, except to state that the eccentricities of near-Earth particles are significant and for that reason we should not expect the zodiacal cloud to be rotationally symmetric with the Sun at the center of symmetry.

To conclude this section, we have demonstrated that we now know how to describe the orbital evolution of a cloud of particles from a given source region, moving under the action of both PR drag, light pressure and gravitational forces.

\section{Modeling the Background Zodiacal Cloud}

Once the structure of the cloud has been specified in terms of (a) the distribution of orbital elements of the dust particle orbits, (b) the distribution of particles on those orbits and (c) the thermal properties of the particles, we need a means of viewing the model cloud and comparing the predicted fluxes with the observations. We have constructed a three-dimensional numerical model (the SIMUL model) that calculates the distribution of flux produced by any particular distribution of dust particle orbits. This model reproduces the exact viewing geometry of the IRAS telescope and allows for the eccentricity of the Earth's orbit. The result is a model for the variation with ecliptic latitude of the brightness observed in a given waveband as the line of sight of the telescope sweeps through the model cloud at a constant elongation angle.

Partly because of the postulated association of the Hirayama asteroid families with the IRAS solar system dustbands (Dermott and Nicholson, 1989), our initial emphasis has been on the dynamical evolution of asteroidal particles, but we do recognize that future models may have to include cometary particles. If the sizefrequency distribution of asteroidal dust is a simple power law, then we expect the effective area of the dust seen in a given waveband $\lambda$ to increase as the particle radii decrease until, at some radius $\lesssim \lambda / 2 \pi$, the absorption coefficient of the particles falls off to zero. Detailed calculations indicate that the flux in the $25 \mu \mathrm{m}$ waveband should

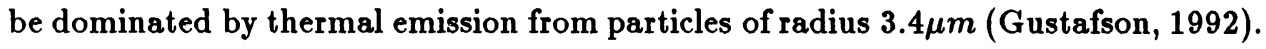
For ease of calculation and heuristic purposes, we assume here that all the particles in the model cloud have that radius.

Predictions for a zodiacal cloud of single-size asteroidal particles that originate in the main belt and have distributions of proper inclinations similar to those of the brighter asteroids but whose semimajor axes decay due to PR drag are shown in Figure 2(b). Because the forced inclinations and nodes vary markedly with heliocentric distance and because Figure 2(b) represents an integrated view of the cloud, the agreement of our model with the IRAS observations is remarkable. However, this does not imply that our model is entirely correct and that no further work 


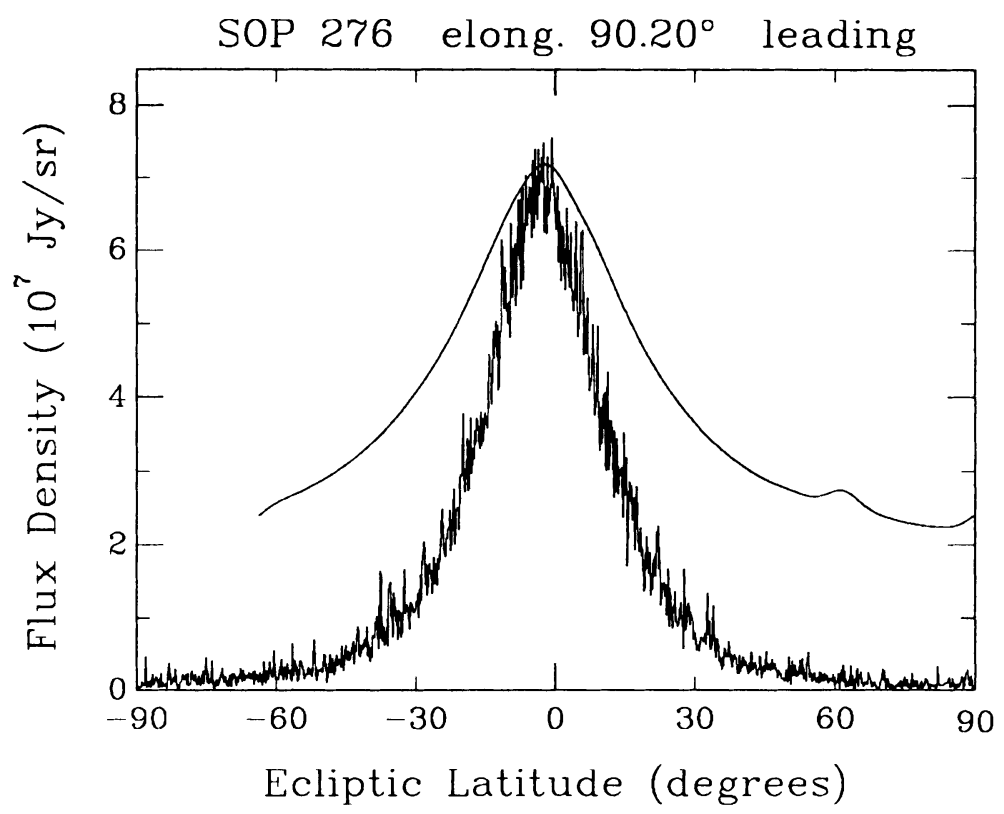

Fig. T. Comparison of a particular IR AS observation (SOP 276 ) of the shape of the zodiacal cloud, that is, the variation of the observed flux in the $25 \mu \mathrm{m}$ waveband with ecliptic latitude for a particular elongation angle $\left(\epsilon=90.2^{\circ}\right)$, with the prediction of a simple asteroidal model based on single-size particles of radii $3.4 \mu \mathrm{m}$.

needs to be done on this problem. The striking agreement may indeed indicate that $3.4 \mu \mathrm{m}$ radii particles, whose orbits are distributed between the asteroid belt and the Sun as dictated by PR drag, have the appropriate distribution of forced inclinations and nodes needed to account for the observed plane of symmetry of the cloud. But there are many other important observations of the cloud for which our model must also account and some of these have proved, at present, to be problematical. In particular, we have found that the single particle-size model does not account for the observed variation of the background flux with ecliptic latitude: the flux in our model at high ecliptic latitudes is far too low (see Figure 7). This is a major discrepancy, but it may have simple remedy. It suggests that we need to analyze model clouds that have heliocentric variations of dust particle size. In particular, clouds of particles whose effective area increases with decreasing heliocentric distance as expected, perhaps, for a cloud of particles that has evolved due to both collisions and PR drag (Durda et al., 1992; Gustafson et al., 1992).

We have commenced work on the combined collisional and orbital evolution of the dust particles and on the determination of their thermal properties (Gustafson, 1992). Figure 8 shows how the area of dust associated with a model Hirayama family decays with time. This calculation is for particles from $1 \mathrm{~mm}$ through the largest asteroidal sizes and describes the stochastic breakup of asteroidal fragments, but does not include PR drag. Extensions to $1 \mu m$ will be made using the full-scale ACE 

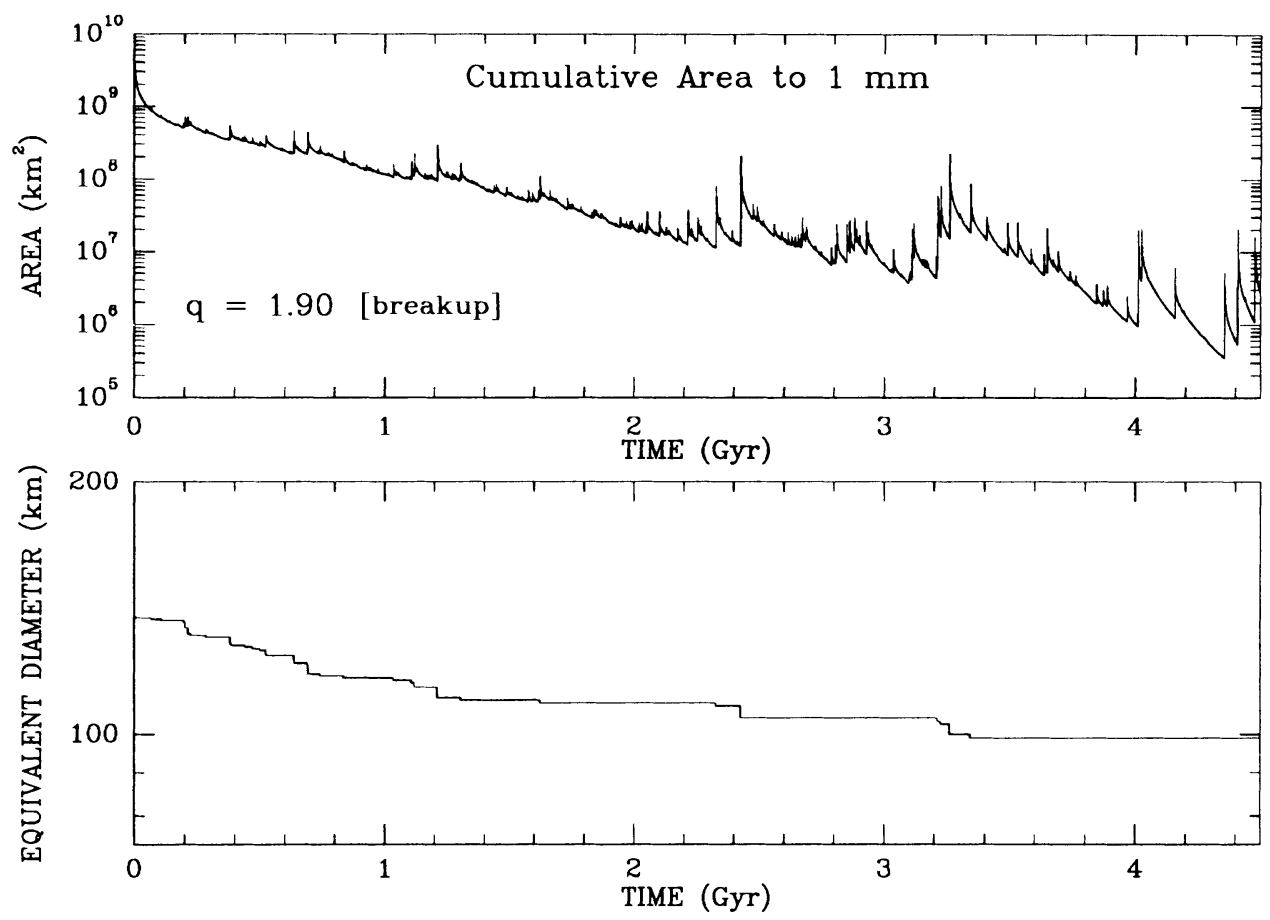

Fig. 8. Variation with time of the total cross-sectional area of dust associated with a Hirayama family for a break-up process with $q=1.90$. The upper plot shows the cumulative area associated with particles of radii $>1 \mathrm{~mm}$. This calculation needs to be extended down to $1 \mu \mathrm{m}$ and we also need to include the effects of PR drag. The lower plot shows the decay of the equivalent diameter of the family.

(Asteroidal Comminute Evolution) code that includes radiation forces and covers 4.5 $10^{9}$ years of collisional and orbital evolution. We have already reported results for calculations spanning the first $310^{7}$ years of evolution that occurs after the break-up of asteroids in the main belt (Gustafson et al., 1992). Figure 8 shows that the dust production rate in the main asteroid belt becomes more stochastic with time following a relatively smooth decrease in area as the small particles created directly from the break-up of the parent body are destroyed. The spikes in the dust production are due to the break-up of small to intermediate size asteroids. Comparison of Figures $8 \mathrm{a}$ and $8 \mathrm{~b}$ illustrates that while the observable volume of the family may decay at fairly constant and well-defined rate, the total area of dust associated with the family during that time may fluctuate by an order of magnitude or more. We have found, however, that the magnitude of the spikes exhibited in Figure 8a is highly sensitive to the exponent $q$ in the power-law size distribution of the collision fragments. The spikes are subdued for values of $q \leq$ 1.837 , the collisional equilibrium value. Thus, our analysis may help distinguish between higher and lower values of $q$. If the dust bands can be shown, conclusively, to be associated with the major Hirayama families, then this will provide statistical 

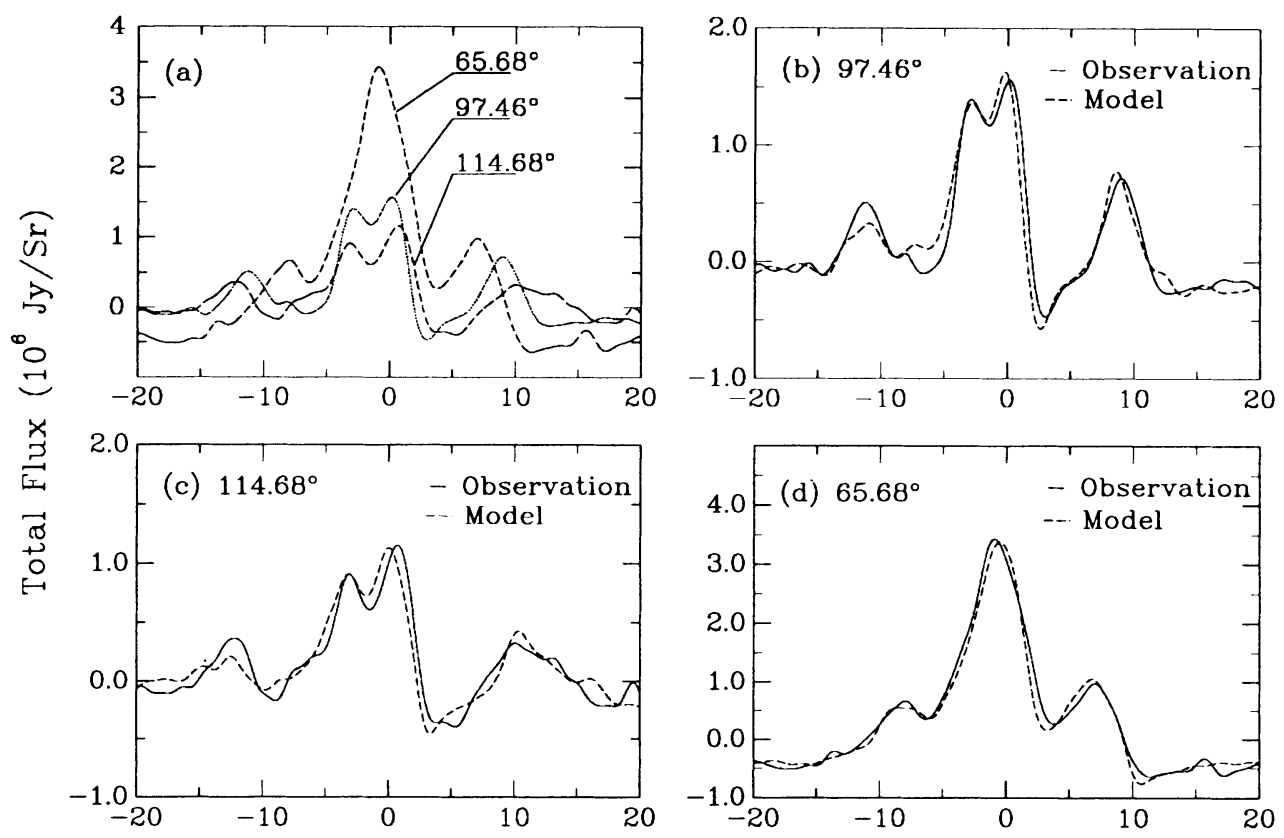

Ecliptic Latitude

Fig. 9. (a) IRAS observations of the dust bands seen at elongation angles $65.68^{\circ} \cdot 97.46^{\circ}$ and $114.68^{\circ}$. Comparisons with model profiles based on the prominent Hirayama asteroid families are shown in (b), (c) and (d).

evidence that asteroidal break-up is not in the highly stochastic, high $q$, regime.

\section{Modeling the Solar System Dustbands}

IRAS observed the solar system dust bands over a wide range of viewing geometries. Using models based on single-sized particles of radii $3.4 \mu \mathrm{m}$ originating in the major Hirayama asteroid families, plus our calculations of the forced orbital elements of these size particles as a function of heliocentric distance and the SIMUL code, we have attempted to account for the shapes, amplitudes and ecliptic latitudes of the dust bands. The three observations shown in Figure $9(\mathrm{a})$ cover nearly the entire range of IRAS elongation angles. They show wide variations in amplitude and shape and the effects of parallax are also clearly evident. These particular profiles are representative of the IRAS data set as a whole and illustrate the highly demanding observational constraints that any model of the dustbands must satisfy.

The model profiles shown in Figures $9(\mathrm{~b}),(\mathrm{c})$ and (d) were generated using dust from five prominent families (Eos, Themis, Koronis and two others). The surface areas associated with the families were treated as free parameters and adjusted to fit the observations at $\epsilon=114.68^{\circ}$. Then, exactly the same particle distributions were used at the other two elongation angles. This procedure was partly successful. 
However, to account for the shape of the 'ten-degree' bands at $\epsilon=65.68^{\circ}$, the area associated with one of the families had to be readjusted. Apart from that discrepancy, the near-perfect fit to the observations, particularly the observations of the central dustbands, may be good evidence that the solar system dust bands are due to the Hirayama families.

With these dust band models, unlike the models for the background cloud, we have been at least partly successful in accounting for the variation of flux with ecliptic latitude, that is, in accounting for the profile shapes. But these results cannot be regarded as conclusive until we have extended our models to include a realistic range of particle sizes. Thus, the status of these results is uncertain. They may constitute very strong evidence that the source of the dust band particles is asteroidal. On the other hand, it is not clear why the same type of model cannot be applied to the asteroid belt as a whole.

We also have evidence in the IRAS observations that dust from the Hirayama families is transported to the Earth by PR-drag. We have shown by modeling that to obtain a central dustband without a marked split with an elongation angle of $65^{\circ}$, a majority of the flux must come from particles between the orbits of Earth and Mars. The good agreement between our models and the observations is also evidence that asteroidal break-up is not in the stochastic regime.

We feel that our success in modeling and interpreting the shape of the dust bands is encouraging, but we also know that the use of a single-size dust particles is oversimplified and is not commensurate with the level of modeling of which we are now capable. We have developed codes to calculate the size and heliocentric distributions of colliding particles subject to radiation forces (ACE) and also ways to convert the size distributions into effective surface areas as a function of heliocentric distance. Because the orbital evolution of dust particles depends on their size, any correct model of the zodiacal emission must allow that the emitted flux is also a function of particle size. The flux depends on the temperature of the dust and its infrared emission efficiency. While there is no consensus on a single model for either asteroidal or cometary particles, we adopt two working-models for asteroidal dust and one for cometary dust. The particles' temperature at a given heliocentric distance is calculated assuming radiative equilibrium with the solar radiation field and re-emission into empty (cold) space. The emitted flux at any wavelength is then given by the Planck function at that temperature times an efficiency factor for the emissivity. This factor is equal to the absorption efficiency and must be obtained at both optical and infrared frequencies to determine the amount of solar energy absorbed (mostly at optical wavelengths) and the amount of energy emitted by the particle in the infrared. Because of the wide frequency range, we are confined to an efficient computational method. In practice, this means the use of Mie theory that is applicable to spherical particles of arbitrary size and refractive indices. The article by Gustafson (1992) describes our models and the use of an effective index of refraction with Mie-theory to represent material consisting of inhomogeneous and aggregated particles. We are also developing a code to calculate the scattering by aggregates more accurately (Gustafson et al., 1992).

When we have an understanding of the collisional and orbital evolution of the particles in the cloud, we will be able to determine the forced orbital elements 
of the dust particles more accurately. This is not simply a problem in celestial mechanics, because interparticle collisions ensure that the sizes of the "particles" do not remain constant as the asteroidal material spirals towards the Sun. Thus, before the forced orbital elements can be determined, we need to know the length of time, on average, that a particle remains at a given size. Much difficult work remains to be done before the cloud can be modeled with confidence, but we have, perhaps, at least made a start in the right direction.

\section{Acknowledgements}

This work is supported by NASA through grants NAG 9 110, NAG 5-1725, and NAGW-1923. It was also supported in part by the University of Florida and the IBM Corporation through their Research Computing Initiative at the Northeast Regional Data Center.

\section{References}

Burns, J. A., Lamy, P. L. and Soter, S. (1979) Radiation Forces on Small Particles in the Solar System. Icarus, 40, 1-48.

Dermott, S. F., Nicholson, P. D., Burns, J. A. and Houck, J. R. (1984) Origin of the Solar System Dust Bands Discovered by IRAS, Nature, 312, 505-509.

Dermott, S. F., Nicholson, P. D., Burns, J. A. and Houck, J. R. (1985) An Analysis of IRAS' Solar System Dust Bands. IAU Colloquium \#85 Properties and Interaction of Interplanetary Dust (eds. R. H. Giese and P. Lamy) D. Reidel Pub. Co., 395-409.

Dermott, S. F., Nicholson (1986) Masses of the Satellites of Uranus. Nature, 319, 115-120.

Dermott, S. F., Nicholson, P. D. and Wolven, B. (1986) Preliminary Analysis of the IRAS Solar System Dust Band Data, In Asteroids, Comets and Meteors, II (eds. C-I. Lagerkvist and H. Rickman), Uppsala, 583-594.

Dermott, S. F., Nicholson, P. D., Kim, Y., Wolven, B. and Tedesco, E. (1988) The Impact of IRAS on Asteroidal Science. In Comets to Cosmology (ed. A. Lawrence), Berlin, Springer Verlag, 3-18.

Dermott, S. F. and Nicholson, P. D. (1989) IRAS Dustbands and the Origin of the Zodiacal Cloud. In Highlights of Astronomy 8, 259-26.

Dermott, S. F., Nicholson, P. D., Gomes, R. S. and Malhotra, R. (1990) Modelling the IRAS Solar System Dustbands. Adv. Space Res. 10, (1)165-(1)172.

Dermott, S. F., Durda, D. D., Gustafson, B. A. S., Jayaraman, S., Xu, Y. L., Gomes, R. S. and Nicholson, P. D. (1992) The Origin and Evolution of the Zodiacal Dust Cloud. In Asteroids, Comets and Meteors, IV (eds. E. Bowell and A. Harris), Flagstaff, in press.

Durda, D. D., Dermott, S. F. and Gustafson, B. A. S. (1992) Modeling of Asteroidal Dust Source Production Rates. In Asteroids, Comets and Meteors, IV, (eds. E. Bowell and A. Harris), Flagstaff, in press.

Flynn G. J. (1992) Large Micrometeorites: Atmospheric Entry Survival Relation to Mainbelt Asteroids, and Implication for the Cometary Dust Flux. In Asteroids, Comets and Meteors, $I V$, (eds. E. Bowell and A. Harris), Flagstaff, in press.

Giese, R. H. and Kneißel, B. (1989) Three-Dimensional Models of the Zodiacal Dust Cloud. II. Compatibility of Proposed Infrared Models. Icarus, 81, 369-378.

Gomes, R. S. and Dermott, S. F. (1992). Icarus, (to be submitted).

Grün E., et al., (1992) Interplanetary Dust Near 1 AU, In Asteroids, Comets and Meteors, IV, (eds, E. Bowell, and A. Harris), Flagstaff, in press.

Gustafson, B. A. S. (1992) Thermal Emission from Asteroidal and Cometary Dust Models. Submitted to Icarus.

Gustafson, B. A. S., Dermott, S. F., Durda, D. D. and Grün, E. (1992) Collisional and Dynamical Evolution of Dust from the Asteroid Belt. In Asteroids, Comets and Meteors, IV, (eds. E. Bowell and A. Harris), Flagstaff, in press. 
Levasseur, A-C. and Blamont, J. (1976) Evidence for Scattering Particles in Meteor Streams. In Interplanetary Dust and Zodiacal Light (eds. H. Elsasser and H. Fechtig), Springer-Verlag, Heidelberg, pp. 58-62.

Schramm, L. S., Brownlee, D., and Wheelock, M. M. (1989) Major Element Composition of Stratospheric Micrometeorites. Meteoritics, 99-112.

Stephens, J. R. and Gustafson, B. A. S. (1991) Laboratory Reflectance Measurements of Analogues to "Dirty" Ice Surfaces on Atmosphereless Solar System Bodies. Icarus, 84, 209-217.

Wyatt, S. P. and Whipple, F. L. (1950) The Poynting-Robertson Effect on Meteor Orbits. Ap. J., 111, 134-141.

\section{Discussion}

H.Kinoshita - Why do you use the ecliptic plane as a reference plane instead of the invariable plane of the solar system?

S.F.Dermott - We need to refer our model calculations to the same plane as that used to describe the observations. Since IRAS orbited the Earth, this plane is the plane of the ecliptic.

J.Štohl - According to Whipple, a main source of the zodiacal cloud particles is the original giant comet P/Encke. Did you try to calculate a model with P/Encke as the source?

S.F.Dermott - We do intend to do similar calculations for cometary particles. However, the secular perturbation of particles in highly eccentric orbits will not be as simple as those of asteroidal particles and it may be that the invariance of the proper inclination does not hold for cometary particles. If that is the case, then we will be able to rule out cometary particles as the source of the dust bands.

H.U.Keller - 1) Do you start out with a 2- or with a 3-dimensional distribution of dust particles? 2) You did not mention any quantitative data for the dust production. Is the model (brightness) in agreement with reasonable dust production by the asteroid families?

S.F.Dermott - The model is fully three dimensional. When we suggested that the Hirayama families are associated with the dust bands in 1985 , we also made estimates of the expected and observed areas and showed that they were in reasonable agreement. More refined calculations are still in progress (Durda and Dermott, Asteroids IV meeting, Flagstaff, 1991), but these have proved to be more difficult than we originally anticipated. 